\title{
Cost-effectiveness of cervical cancer screening with primary human papillomavirus testing in Norway
}

\author{
EA Burger', JD Ortendahl' ${ }^{2}$, S Sy ${ }^{2}$, IS Kristiansen' and JJ Kim ${ }^{*, 2}$ \\ 'Department of Health Management and Health Economics, University of Oslo, PO BOX 1089 , Oslo 01 37, Norway; ${ }^{2}$ Department of Health Policy and \\ Management, Center for Health Decision Science, Harvard School of Public Health, 718 Huntington Avenue, 2nd Floor, Boston, MA 02I I5, USA
}

\begin{abstract}
BACKGROUND: New screening technologies and vaccination against human papillomavirus (HPV), the necessary cause of cervical cancer, may impact optimal approaches to prevent cervical cancer. We evaluated the cost-effectiveness of alternative screening strategies to inform cervical cancer prevention guidelines in Norway.

METHODS: We leveraged the primary epidemiologic and economic data from Norway to contextualise a simulation model of HPVinduced cervical cancer. The current cytology-only screening was compared with strategies involving cytology at younger ages and primary HPV-based screening at older ages (31/34 + years), an option being actively deliberated by the Norwegian government. We varied the switch-age, screening interval, and triage strategies for women with HPV-positive results. Uncertainty was evaluated in sensitivity analysis.

RESULTS: Current cytology-only screening was less effective and more costly than strategies that involve switching to primary HPV testing in older ages. For unvaccinated women, switching at age 34 years to primary HPV testing every 4 years was optimal given the Norwegian cost-effectiveness threshold (\$83000 per year of life saved). For vaccinated women, a 6-year screening interval was cost-effective. When we considered a wider range of strategies, we found that an earlier switch to HPV testing (at age 31 years) may be preferred.

CONCLUSIONS: Strategies involving a switch to HPV testing for primary screening in older women is expected to be cost-effective compared with current recommendations in Norway.

British Journal of Cancer (2012) I 06, I57I - 1578. doi:I0.1038/bjc.2012.94 www.bjcancer.com
\end{abstract}

Published online 22 March 2012

(c) 2012 Cancer Research UK

Keywords: cost-effectiveness; cervical cancer screening; HPV vaccination

Cytology-based screening programmes that have achieved comprehensive coverage have been credited with significant reductions in incidence of and mortality from invasive cervical cancer through early detection and treatment (Hakama and Hristova, 1997; Peto et al, 2004; Bray et al, 2005). Despite successful screening, cervical cancer is still among the three most frequent cancers for women 25-49 years of age in Norway, where incidence and mortality rates are 9.5 and 1.7 per 100000 women-years, respectively (Cancer Registry of Norway, 2011). Since 1995, the Norwegian Coordinated Cervical Cancer Screening Program has invited women to cytology-based screening every 3 years. Recent clinical studies have reported that human papillomavirus (HPV) DNA testing has a higher sensitivity for detecting high-grade precancerous lesions (Arbyn et al, 2006), possibly resulting in more opportunities for early detection and treatment. In addition, data combined from six European countries suggests that the primary screening interval may be safely extended by using HPV DNA testing (Dillner et al, 2008).

In the autumn of 2009, vaccination against HPV was introduced as part of the childhood immunisation programme for preadoles-

*Correspondence: Dr JJ Kim; E-mail: jkim@hsph.harvard.edu Received 21 November 2011; revised 17 February 2012; accepted 23 February 2012; published online 22 March 2012 cent girls, free of charge. The HPV vaccine protects against two carcinogenic HPV types, 16 and 18, that cause $\sim 70 \%$ of cervical cancers in Norway, as well as two non-carcinogenic types, 6 and 11 , that cause the majority of genital warts. HPV vaccination of older women has not been implemented, and screening will continue to remain the main source of prevention against cervical cancer for the current population of Norwegian women who are past the vaccination target age, as well as those who do not receive the vaccine in adolescence. Importantly, screening will also continue to be critical among those vaccinated to prevent the $30 \%$ of cancer cases that are not attributable to the vaccine types.

Given the availability of HPV vaccines and highly sensitive HPV DNA tests (Franco, 2003; Arbyn et al, 2006; Cuzick et al, 2006a, b), countries around the world are evaluating new screening algorithms that use HPV DNA testing for primary screening; however, determining the optimal approach to cervical cancer prevention is quite complex and involves multiple tradeoffs. For example, despite the higher sensitivity of the HPV DNA test for high-grade cervical intraepithelial neoplasia (CIN), health officials have concerns with regard to the low clinical specificity of the test, which may result in over referrals (i.e., excess burden for women and health services). This limitation may be minimised by an algorithm that relies more heavily on identifying HPV persistence rather than immediately subjecting women directly to colposcopy/ biopsy, a diagnostic procedure that may be associated with 
increased anxiety compared with routine testing. The use of decision-analytic methods to synthesise and extrapolate clinical, epidemiological, and economic evidence beyond the capacity of empirical trials, may aid decisions regarding the optimal secondary prevention strategies under various scenarios of uncertainty (Goldie, 2003). Such models can estimate the lifetime risk of dying from cervical cancer, life expectancy, and lifetime costs related to screening and treatment of cervical cancer. These data are then used to estimate the additional costs and life years saved of a particular screening strategy, compared the current recommended strategy. In Norway, the health benefit of an intervention or strategy is considered to be good value for money if the additional life year costs $<500000$ Norwegian Kroner (NOK; $\approx \$ 83000$ ). In this study, we use a decision-analytic model to assess the impact of adopting recently proposed cervical cancer prevention strategies involving primary HPV DNA testing (Cancer Registry of Norway, 2011) in order to inform policy recommendations in Norway. Specifically, our analysis addresses whether women who have been vaccinated against HPV can be screened efficiently using primary HPV DNA testing and whether the optimal strategy may differ for those women who have not been vaccinated.

\section{MATERIALS AND METHODS}

\section{Analytic approach}

We adapted an existing mathematical model to reflect the natural history of HPV-induced cervical cancer in Norway (GoldhaberFiebert et al, 2007; Kim et al, 2007; Kim and Goldie, 2008). The model was adjusted to the Norwegian context using primary clinical and cost data from Norway to project the health and economic outcomes associated with different scenarios of screening. We compared the currently recommended cytology-based programme with alternative screening strategies that use HPV DNA testing for women who have been either vaccinated or not vaccinated against HPV-16 and HPV-18 in pre-adolescence. Outcomes included lifetime risk of cancer, life expectancy, and lifetime costs. Incremental cost-effectiveness ratios (ICERs), calculated as the additional dollar (\$) for each additional year of life saved (YLS) of a strategy compared with the next most costly strategy, was used as a performance indicator. Strategies that were more costly and less effective (dominated) or less costly and less cost-effective (weakly dominated) were removed from the costeffectiveness calculations. The 'most cost-effective' intervention is not necessarily the one that has the lowest ratio as society may be willing to pay more for health benefit. We used the proposed willingness-to-pay threshold of $500000 \mathrm{NOK}(\approx \$ 83000)$ per YLS to signify the amount below which an intervention would be considered 'good value for money' (Norwegian Directorate of Health, 2007). We adopted a societal prospective, including all costs and benefits regardless to whom they accrue, and discounted costs and benefits by $4 \%$ per year, as recommended in Norway (Norwegian Finance Department, 2005). Uncertain parameters and scenarios were explored extensively in sensitivity analysis.

\section{Model overview}

The individual-based stochastic model has been previously described (Goldhaber-Fiebert et al, 2007; Kim et al, 2007; Kim and Goldie, 2008). The model simulates the natural history of HPV-induced cervical cancer in a series of mutually exclusive, collectively exhaustive health states. A cohort of females enters the model and can transition between health states in monthly intervals until death. Transition probabilities are a function of HPV type, history of prior HPV infection (i.e., natural immunity), and age. The model is static in that HPV incidence changes as a function of age, but does not change as a function of sexual activity or HPV prevalence in the population over time. Indirect effects of vaccination on risk of HPV infection (i.e., herd immunity) were explored in a sensitivity analysis by reducing HPV incidence in unvaccinated women. Health states were stratified according to HPV infection (no infection, high-risk type 16, high-risk type 18, other high-risk types and low-risk types (Munoz et al, 2003)), CIN grade 1 (CIN1), CIN grade 2, 3 (CIN23), invasive cancer (local, regional or distant), and death. Women with cancer can be identified either through screening or from symptoms, or they may remain undetected and progress to more advanced stages of cancer. Women with cancer face stage-specific survival rates; all women face competing mortality risks from other causes based on Norway life tables (Statistics Norway, 2011).

\section{Epidemiologic data}

Baseline transition parameter values describing the natural history of disease were based on the best available empirical data and assume that the underlying mechanism of cervical carcinogenesis does not vary across epidemiological settings. However, risk factors, such as sexual behaviour, and cervical cancer incidence rates differ between countries; therefore, country-specific data are needed to adjust baseline inputs to account for variations in progression and regression rates. We leveraged empirical data from Norway and used a likelihood-based algorithm to identify candidate sets of parameter values that achieve good-fit to epidemiological outcomes observed in the Norwegian population. Specifically, Norwegian data used for calibration included agespecific prevalence of HPV-16, HPV-18 (Mari Nygaard, personal communication) and of CIN23 in Norwegian women (Molden et al, 2005, 2006); the relative contributions of HPV-16, HPV-18 and other high-risk HPV types in CIN23 and cervical cancer (Steinar Thoresen, personal communication), and pre-screening (19531969) cancer incidence rates were obtained from the Cancer Registry of Norway. The parameterisation and likelihood-based calibration process have been described previously (GoldhaberFiebert et al, 2007; Kim et al, 2007; Kim and Goldie, 2008); details of the Norwegian-specific calibration for the current analysis are included in the Supplementary Appendix. All analyses were conducted with 50 statistically indistinguishable (i.e., good-fitting) parameter sets to incorporate the effect of uncertainty surrounding the natural history of cervical cancer. Results were reported as the mean of outcomes across the 50 parameter sets, and ICERs were calculated as the incremental mean costs divided by the incremental mean effects of two strategies (Stinnett and Paltiel, 1997). Screening test characteristics (i.e., sensitivity and specificity) were based on published studies and varied in sensitivity analyses (Franco, 2003; Sherman, 2003; Solomon, 2003; Arbyn et al, 2006; Cuzick et al, 2006a, b).

\section{Cost data}

Direct medical and non-medical costs associated with screening, vaccination, and treatment were estimated using a combination of official Norwegian guidelines (Norwegian Medical Association, 2010a) and expert opinion. All costs were measured in $2010 \mathrm{NOK}$ and converted to US dollars (US \$) using the average annual 2010 exchange rate (US $\$ 1=$ NOK6.05) (Federal Reserve, 2011). Direct medical costs for screening, diagnosis, and treatment of dysplasia and invasive cervical cancer were based on Norwegian Diagnosis Related Groups (DRGs) and the Fee Schedules for General Practitioners and Specialists (Norwegian Directorate of Health, 2010; Norwegian Medical Association, 2010b, c; Table 1). Screening laboratory costs were adjusted to reflect potential discrepancies between published reimbursement rates and true economic costs (additional details in the Supplementary Appendix). We based HPV vaccination costs on a published report from the Norwegian 
Table I Selected model inputs

\begin{tabular}{|c|c|c|}
\hline Cost & $\begin{array}{l}\text { Value } \\
\text { (\$) }\end{array}$ & $\begin{array}{c}\text { Range } \\
(\$)\end{array}$ \\
\hline \multicolumn{3}{|l|}{ Screening } \\
\hline Conventional cytology & 49 & $8^{\mathrm{a}}$ \\
\hline Liquid-based cytology & 50 & $12^{\mathrm{a}}$ \\
\hline HPV DNA testing ${ }^{\mathrm{ot}}$ & 62 & $54^{\mathrm{a}}$ \\
\hline Office visit, patient time, and transport & 160 & $80-321$ \\
\hline Colposcopy with biopsy ${ }^{c}$ & 337 & $168-674$ \\
\hline \multicolumn{3}{|l|}{ CIN treatment ${ }^{c}$} \\
\hline $\mathrm{CINI}$ & 1024 & $512-2047$ \\
\hline $\mathrm{CIN} 2 / 3$ & 2162 & $1081-4325$ \\
\hline \multicolumn{3}{|l|}{ Cancer treatment ${ }^{\mathrm{c}}$} \\
\hline Local & 25770 & $12885-51539$ \\
\hline Regional & 51589 & $25795-10317$ \\
\hline Distant & 59635 & $29818-119270$ \\
\hline \multicolumn{3}{|l|}{ Vaccine } \\
\hline Per dose & 163 & \\
\hline Test characteristics & $\begin{array}{c}\text { Value } \\
\text { (\%) }\end{array}$ & $\begin{array}{c}\text { Range } \\
\text { (\%) }\end{array}$ \\
\hline \multicolumn{3}{|l|}{ HPV DNA performance for detection of $C I N^{d}$} \\
\hline Probability of HR-HPV given HR-HPV & 100 & \\
\hline Probability of no HR-HPV given no HR-HPV & 100 & \\
\hline \multicolumn{3}{|l|}{ Cytology performance for detection of $\mathrm{CIN}^{\mathrm{e}}$} \\
\hline Probability of abnormal cytology given CINI & 70 & $40-70$ \\
\hline Probability of abnormal cytology given $\mathrm{CIN} 2 / 3+$ & 80 & $40-80$ \\
\hline $\begin{array}{l}\text { Probability of normal cytology given } \\
\text { normal histology }\end{array}$ & 95 & \\
\hline
\end{tabular}

Abbreviations: HPV = human papillomavirus; $\mathrm{CIN}=$ cervical intraepithelial neoplasia.

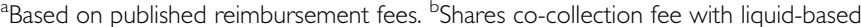
cytology. Includes office costs, patient time, and transport. ${ }^{\text {DP }}$ Probability of HR-HPV DNA positivity given high-risk HPV is assumed to be $100 \%$; output from the model indicates the clinical sensitivity of HPV DNA testing for detecting CIN2 or worse is 80\% (min: 62\%; max: 95\%). Specificity for CIN2 and worse is 89\% (min: 85\%; max: $94 \%) .{ }^{e}$ Abnormal cytology is defined as atypical squamous cells of undetermined significance or worse. Local: stage la-Ila; Regional: stage Ilb-IIlb; Distant: IVa-IVb. All costs are expressed in 2010 US dollars (US $\$=$ NOK6.05).

Medicines Agency and assumed a three-dose regimen administered over the course of 6 months (Norwegian Medicines Agency, 2010).

Direct non-medical costs were estimated to account for the production loss and the transportation costs associated with screening and treatment. We used the average 2010 gross monthly income of Norwegian women obtained from Statistics Norway (2011) and adjusted the wage to include social benefits paid by employers. Travel time and transportation costs associated with screening and follow-up visits were estimated from a prospective study of colorectal screening in Norway (Aas, 2009). The time spent travelling to a hospital to receive cervical cancer treatment was estimated from a health survey conducted by the Statistics Norway for the World Health Organization (2003). We attributed zero production loss or transportation costs for the children or their parents to receive the HPV vaccination, as it is given as part of the school administered vaccination programme for girls in the 7 th grade. Additional details and costing assumptions can be found in the Supplementary Appendix. The costs of cancer treatment, CIN treatment, screening test, colposcopy, and office visits were varied widely (50 and $200 \%$ of base case values) in oneway sensitivity analyses.

\section{Strategies}

The current Norwegian screening strategy involves triennial cytologic evaluation of cervical cells (i.e., cytology) followed by repeat cytology in combination with HPV testing for atypical squamous cells of undetermined significance or low-grade squamous intraepithelial lesion (LSIL) 6 months later (herein referred to as 'cytology-based screening'). Women with high-grade squamous intraepithelial lesions are referred directly to colposcopy/biopsy. The proposed strategy involves switching older women (age $\geqslant 34$ years) from the current strategy to primary HPV DNA testing with liquid-based cytology (LBC) triage for women found to be positive for hrHPV types (herein referred to as 'HPV with reflex LBC'). For women who are HPV-positive and cytologynegative (HPV $+/ \mathrm{Cyt}-$ ), the strategy uses a period of intensified screening to identify women who are persistently HPV + /Cyt-, requiring three additional $\mathrm{HPV}+/ \mathrm{Cyt}-$ results each with 12 months apart before receiving a referral for colposcopy/biopsy.

Our primary analysis included 24 variations of the strategy specified by the Norwegian proposal and immediately relevant for Norwegian policy decisions (Figure 1). We compared variations of the strategy that differed by routine screening interval, the number of persistent HPV + /Cyt- results, and the month interval between repeat testing required prompting colposcopy. We then conducted a secondary analysis, which included the same strategies but allowed the age at which women switch from cytology to primary HPV testing (i.e., 31 or 34 years) to vary. In addition, we allowed younger women with LSIL to be referred directly to colposcopy, as recommended in other settings (Wright et al, 2007). The secondary analysis also included a strategy of pre-adolescent vaccination only without screening. For all analyses, we held the screening start and stop ages constant and maintained the 3-year screening interval for younger women (pre-switch). We evaluated the optimal strategies for two sub-groups of women: those who had been vaccinated and those who had not been vaccinated in pre-adolescence.

Screening compliance was assumed to be $100 \%$ to allow comparison of the maximum benefit for each strategy; however, this assumption was varied in sensitivity analysis. For this variation, we assumed that the risk is equally distributed across attenders and non-attenders and there was no change in future screening behaviour. For strategies that incorporate vaccination, we assumed that: (1) the vaccination is given to sexually naive girls at the age of 12 years; (2) all girls receive the recommended three doses of the vaccine; (3) vaccination is $100 \%$ effective in preventing HPV-16, HPV-18, but does not give any protection against contracting other high-risk HPV types (i.e., no crossprotection); and (4) duration of vaccine immunity is lifelong (see Supplementary Appendix for additional assumptions).

In addition to varying costs, we varied the test characteristics of cervical cytology and evaluated the impact of uncertainty around herd immunity, vaccine efficacy, and waning vaccine protection in sensitivity analyses. To explore the impact of screening coverage, we applied a distribution of screening frequencies across the cohort. For this we assumed $15 \%$ were non-screeners, $70 \%$ complied with the specified interval, and the remaining $15 \%$ were screened less frequently (1 year delay each screening round). Although simplified, these assumptions are consistent with estimates documented by the Norwegian Cancer Registry (Cancer Registry of Norway, 2009). Last, we conducted a probabilistic sensitivity analysis by using the 50 good-fitting parameter sets.

\section{RESULTS}

\section{Analysis including currently proposed Norwegian strategies only}

In the primary analysis and regardless of vaccination status, the current cytology-based screening strategy was less effective and 


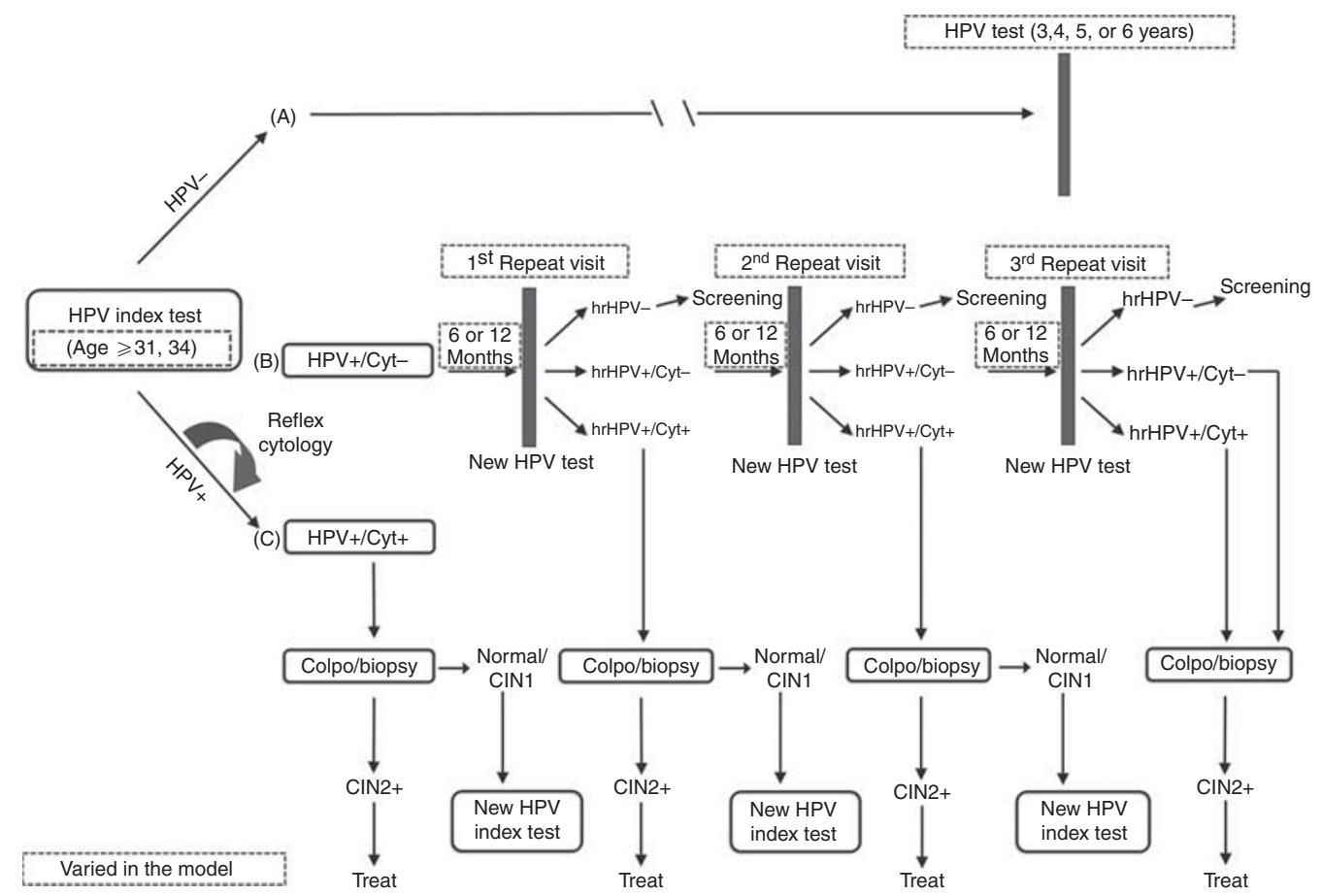

Figure I Flow diagram for proposed HPV DNA screening strategy. The strategy involves switching older women (age $\geqslant 31$ or 34 years) from cytologybased screening to primary HPV DNA testing with LBC triage for women found to be positive for hrHPV types; women with abnormal cytology are then referred directly to colposcopy with biopsy. We compared variations of the strategy, which differed by screening interval (3-6 years), the number of persistent HPV + /Cyt- results (e.g., I, 2, or 3 persistent result(s)), and the month interval between repeat testing (e.g., 6- or I2-month follow-up intervals) required to prompt colposcopy. In the base case, the switch age of screening was 34 years; in a secondary analysis, we allowed women to switch at an earlier age (3I years). Abbreviations: $\mathrm{CIN}=$ cervical intraepithelial neoplasia; Colpo/biopsy = colposcopy with biopsy; hrHPV = high-risk human papillomavirus; HPV + /Cyt- = HPV-positive and cytology-negative result; LBC = liquid-based cytology.

more costly (i.e., strongly dominated) than proposed strategies that involve switching to primary HPV testing at 34 years of age. For unvaccinated women, the optimal (cost-effective) strategy involves switching at age 34 years to primary HPV DNA testing with a 4-year screening interval. For women HPV + /Cyt-, optimal management involves three additional persistent HPV +/Cytresults 6 months apart, before colposcopy referral. This strategy is associated with a cost-effectiveness ratio of $\$ 83000$ per YLS, compared with the next best strategy and yields an expected reduction in lifetime cervical cancer risk of $65 \%$, compared with no screening (Figure 2A). By comparison, the current cytology-based screening programme gives an expected cancer risk reduction of $\sim 55 \%$.

For vaccinated women, the preferred screening strategy involves extension of the screening interval to every 6 years after the switchage of 34 years with the same follow-up of HPV + /Cyt- women as for unvaccinated women. This strategy had a cost per YLS of $\$ 76000$, compared with the next best strategy and an expected cancer risk reduction of $85.7 \%$ (Figure 2B); nearly the same expected reduction as screening women every 3 years with the current strategy, but for a lower lifetime cost. If vaccinated women followed the same strategy that is optimal for unvaccinated women (i.e., screening every 4 years after age 34 years), the ICER would be well over what is considered good value for the cost. On the other hand, if older, unvaccinated women were screened every 6 years (as recommended for vaccinated women), rather than every 4 years, they would be forgoing an additional $8 \%$ absolute reduction in cancer, compared with no intervention.

\section{Analysis including additional strategies}

Switching unvaccinated women to primary HPV DNA testing at age 31 years was always preferred over strategies that involved switching at the proposed age of 34 years. Switching at a younger age provided equal or greater reductions in cervical cancer and could cost up to $24 \%$ less over a woman's lifetime compared with the current screening strategy (Table 2). The optimal strategy, with an ICER of $\$ 76000$ per YLS, entails switching at age 31 years to primary HPV DNA testing every 4 years with reflex LBC (Table 2; top panel). This strategy requires women who are HPV + /Cyt- to have three persistent results 12 months apart, before colposcopy referral. This strategy, compared with the optimal strategy, identified by our primary analysis has similar benefits, but would be expected to cost $\sim 5 \%$ less per woman over her lifetime. Variants of this screening strategy were less attractive. If a 3-year screening interval was maintained for older women using HPV DNA testing and the most intensive follow-up of HPV + /Cyt- women, we estimated that it provides nominal life expectancy gains at a cost of approximately $\$ 513000$ per YLS, compared with the next best strategy.

For women vaccinated during adolescence, switching at an earlier age to HPV DNA testing may also provide similar benefit at a lower cost per woman compared with switching at age 34 years. The optimal strategy involved a 6-year screening interval after the switch-age of 31 years and requires two additional HPV + /Cytresults 12 months apart, before colposcopy referral (Table 2; bottom panel). Compared with switching women to 6-yearly HPV screening at age 34 years and the current cytology-based programme, vaccinated women may achieve similar cancer risk reductions by switching at the earlier age but could reduce the cost per woman over her lifetime by an additional $5 \%$ and $18 \%$, respectively.

\section{Sensitivity analysis}

Overall results were not sensitive to cancer and CIN treatment costs or the imperfect screening compliance scenarios. Results 
$\checkmark$ with $L B C$ triage

(3 years), 1 additional
HPV+/Cyt- 6 months

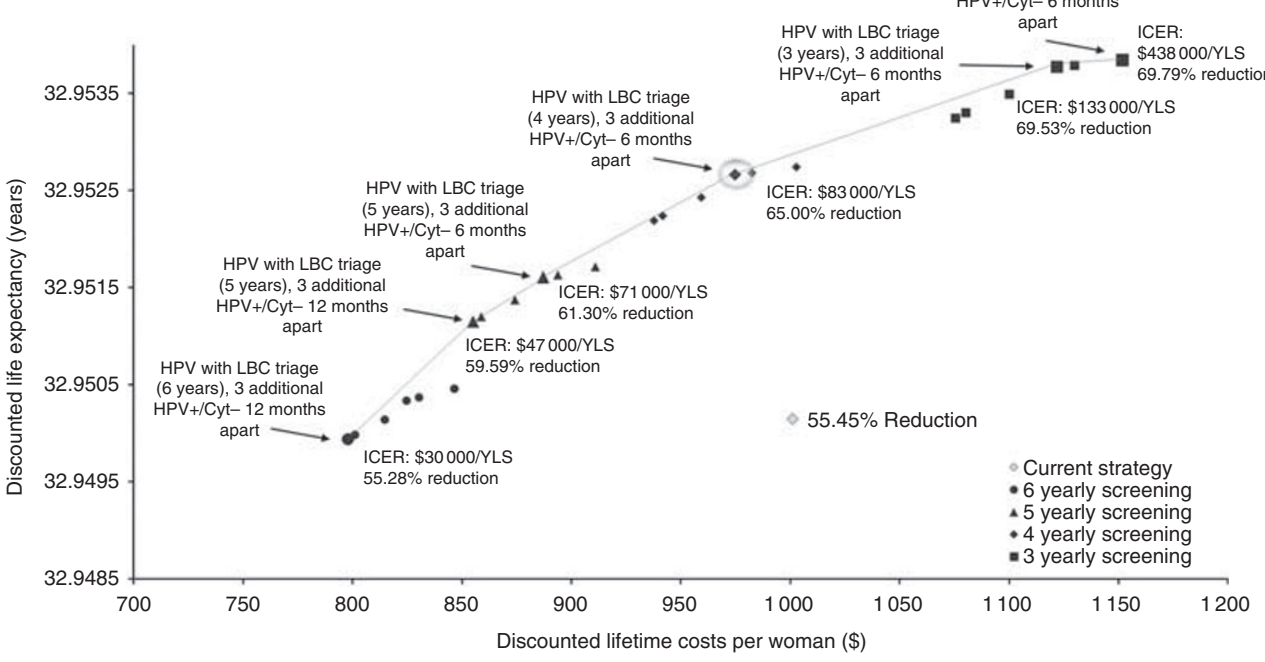

B

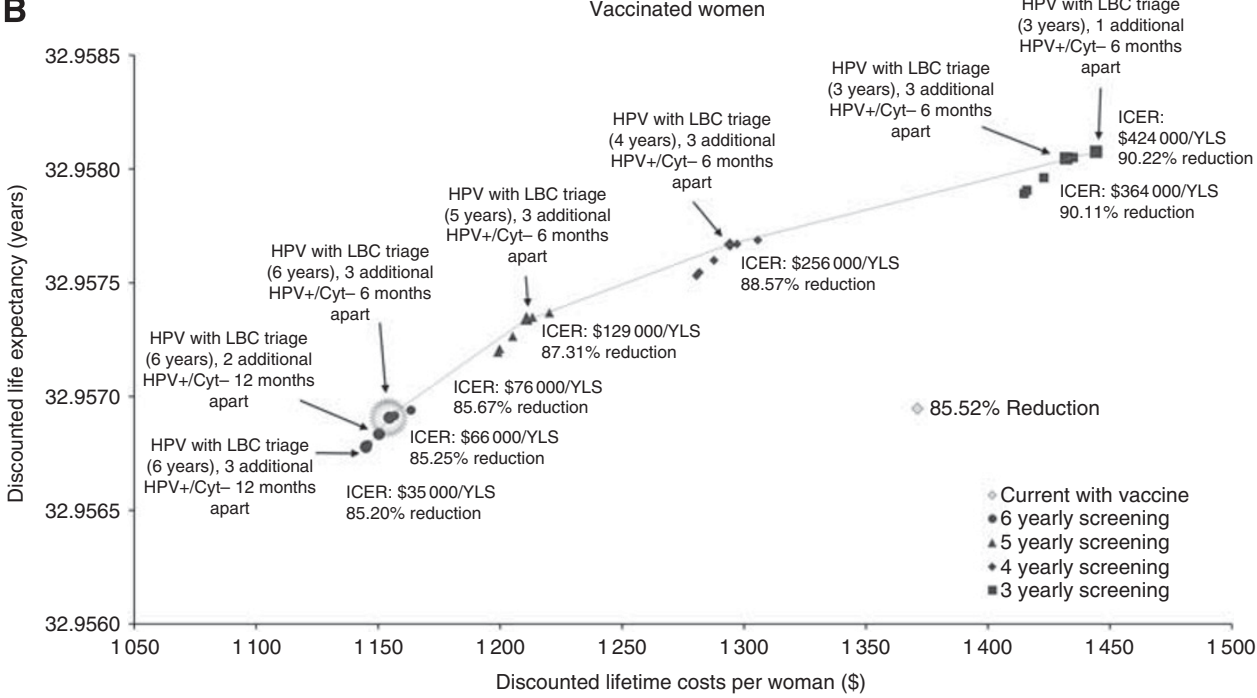

Figure 2 Efficiency frontiers showing the trade-off of costs and benefits. Discounted life expectancy, lifetime costs, reduction in lifetime risk of cancer, and ICERs for alternate cervical cancer screening strategies for women 34 years and older from the 'primary analysis' (see the Results for details) for either unvaccinated $(\mathbf{A})$ or vaccinated (B) women. Strategies lying on the efficiency curve are either less costly and more effective (i.e., strongly dominant) or more costly but more cost-effective (i.e., weakly dominant) than those lying to the right of the curve. The slope of the efficiency curve (also the inverse of the ICER) will be steeper when the net gain in the life expectancy per dollar is greater. Abbreviations: HPV = human papillomavirus; HPV + /Cyt- = HPVpositive and cytology-negative result; ICER = incremental cost-effectiveness ratio; LBC = liquid-based cytology.

were moderately influenced by screening costs, colposcopy costs, and vaccine efficacy. For example, if the cost of a colposcopy/ biopsy doubled ( $\$ 674$ rather than the $\$ 337$ assumed in the base case), the optimal primary screening interval for vaccinated women remained constant, but the follow-up strategy requires three persistent HPV +/Cyt- results rather than two persistent $\mathrm{HPV}+/$ Cyt - results to prompt referral for colposcopy. Results were most sensitive to cytology test characteristics, inclusion of herd immunity benefits, and office-visit costs. When we explored the potential decrease in the sensitivity of cytology after the introduction of vaccination, the most cost-effective strategy for vaccinated women require fewer persistent $\mathrm{HPV}+/ \mathrm{Cyt}-$ results before verification using colposcopy/biopsy. Simulating the indirect protection unvaccinated women receive from vaccinated women allowed for extension of the recommended screening interval by 1 year (i.e., every 5 years rather than every 4 years for women 31 years and older). If office-visit costs doubled (including the transport and time costs of women), it was optimal to refer women to colposcopy/biopsy after only one persistent $\mathrm{HPV}+$ I
Cyt- result rather than requiring three persistent results. Additional sensitivity analysis results are included in the Supplementary Appendix.

We used the 50 good-fitting natural history parameter sets to estimate the probability that the optimal strategies in the primary analysis are cost-effective according the Norwegian cost-effectiveness threshold. For unvaccinated women 34 years or older, switching to primary HPV DNA testing with a 4-year screening interval was found optimal in the majority of the simulations $(58 \%)$, whereas a 6 -year screening interval was never preferred. The analogous results for vaccinated women indicated that a 6year screening interval was optimal in $94 \%$ of the simulations and a 5 -year screening interval was optimal in $6 \%$ of the simulations.

\section{DISCUSSION}

With the advent of new HPV diagnostics, secondary preventative strategies have the potential to further reduce the burden of 
Table 2 Cost-effectiveness results for the analysis including additional strategies

\begin{tabular}{|c|c|c|c|c|c|c|c|c|c|c|c|c|}
\hline $\begin{array}{l}\text { Screening } \\
\text { start age }\end{array}$ & $\begin{array}{l}\text { Screening } \\
\text { frequency, } \\
\text { pre-switch } \\
\text { (years) }\end{array}$ & $\begin{array}{l}\text { Primary } \\
\text { screening } \\
\text { test, } \\
\text { pre-switch }\end{array}$ & $\begin{array}{c}\text { Screening } \\
\text { switch } \\
\text { age }\end{array}$ & $\begin{array}{c}\text { Screening } \\
\text { frequency, } \\
\text { post-switch } \\
\text { (years) }\end{array}$ & $\begin{array}{l}\text { Primary } \\
\text { screening } \\
\text { test, } \\
\text { post-switch }\end{array}$ & $\begin{array}{l}\text { Wait } \\
\text { time for } \\
\text { rescreen } \\
\text { HPV+/Cyt- } \\
\text { (months) }\end{array}$ & $\begin{array}{l}\text { No. of } \\
\text { additional } \\
\text { HPV+/Cyt- } \\
\text { results to } \\
\text { colposcopy }\end{array}$ & Vaccine & $\begin{array}{l}\text { Absolute } \\
\text { reduction in } \\
\text { cancer (\%) }\end{array}$ & $\begin{array}{c}\text { Total } \\
\text { cost per } \\
\text { woman }(\$)^{\mathrm{a}}\end{array}$ & $\begin{array}{l}\text { Total } \\
\text { LE }^{\mathrm{a}}\end{array}$ & $\begin{array}{c}\text { ICER } \\
\text { (\$/YLS) }\end{array}$ \\
\hline \multicolumn{13}{|c|}{ Unvaccinated women } \\
\hline - & - & None & - & - & - & - & - & No & - & 120 & 32.9276 & - \\
\hline 25 & 3 & Cytology ${ }^{b}$ & None & None & None & $6^{c}$ & $I^{c}$ & No & 55.45 & $100 \mid$ & 32.9502 & Dominated \\
\hline 25 & 3 & Cytology ${ }^{b}$ & 31 & 6 & HPV & 12 & 3 & No & 55.59 & 760 & 32.9500 & 29000 \\
\hline 25 & 3 & Cytology ${ }^{b}$ & 31 & 5 & HPV & 12 & 3 & No & 58.82 & 822 & 32.9510 & 57000 \\
\hline 25 & 3 & Cytology & 31 & 4 & HPV & 12 & 3 & No & 63.44 & 922 & 32.9524 & 76000 \\
\hline 25 & 3 & Cytology & 31 & 4 & HPV & 6 & 3 & No & 65.26 & 971 & 32.9529 & 98000 \\
\hline 25 & 3 & Cytology ${ }^{d}$ & 31 & 4 & HPV & 6 & 3 & No & 65.39 & 982 & 32.9529 & 121000 \\
\hline 25 & 3 & Cytology ${ }^{d}$ & 31 & 3 & HPV & 6 & 3 & No & 70.22 & 1160 & 32.9542 & 144000 \\
\hline 25 & 3 & Cytology $^{d}$ & 31 & 3 & HPV & 6 & I & No & 70.49 & 1200 & 32.9543 & 513000 \\
\hline \multicolumn{13}{|c|}{ Vaccinated women } \\
\hline- & - & None & - & - & - & - & - & No & - & 120 & 32.9276 & - \\
\hline- & - & None & - & - & - & - & - & Yes & 63.54 & 646 & 32.9490 & 17000 \\
\hline 25 & 3 & Cytologyb & None & None & None & $6^{c}$ & $1^{c}$ & Yes & 85.52 & $|54|$ & 32.9569 & Dominated \\
\hline 25 & 3 & Cytology ${ }^{b}$ & 31 & 6 & HPV & 12 & 2 & Yes & 85.38 & 1267 & 32.9568 & 80000 \\
\hline 25 & 3 & Cytologyb & 31 & 6 & HPV & 6 & 3 & Yes & 85.81 & 1279 & 32.9570 & 92000 \\
\hline 25 & 3 & Cytologyb & 31 & 5 & HPV & 6 & 3 & Yes & 86.89 & 1339 & 32.9573 & 185000 \\
\hline 25 & 3 & Cytology ${ }^{b}$ & 31 & 4 & HPV & 6 & 3 & Yes & 88.48 & 1439 & 32.9577 & 229000 \\
\hline 25 & 3 & Cytologyb & 31 & 4 & HPV & 6 & 2 & Yes & 88.50 & 1442 & 32.9577 & 390000 \\
\hline 25 & 3 & Cytologyb & 31 & 3 & HPV & 6 & 3 & Yes & 90.25 & 1609 & 32.9581 & 418000 \\
\hline 25 & 3 & Cytologyb & 31 & 3 & HPV & 6 & I & Yes & 90.36 & 1625 & 32.9582 & 544000 \\
\hline 25 & 3 & Cytology ${ }^{d}$ & 31 & 3 & HPV & 6 & I & Yes & 90.39 & 1636 & 32.9582 & 707000 \\
\hline
\end{tabular}

Abbreviations: HPV = human papillomavirus; LE = discounted life expectancy; ICER = incremental cost-effectiveness ratio; HPV+/Cyt-: HPV-positive, cytology-negative result. ${ }^{a}$ Discounted at $4 \%$ per year. All costs are expressed in 2010 US dollars (US $\$=$ NOK6.05). ${ }^{b}$ Combo test triage (HPV/cytology) 6 months later for atypical squamous cells of undetermined significance (ASCUS) and low-grade squamous intraepithelial lesion results. ${ }^{\mathrm{C}} \mathrm{Held}$ constant for all combo strategies for younger women. ${ }^{\mathrm{d} C o m b o}$ test triage (HPV/cytology) 6 months later for ASCUS results only.

cervical cancer. For countries that have implemented the HPV vaccination, two distinct risk groups will emerge as cohorts of vaccinated girls become eligible for screening. Model-based analyses can assist decision-makers faced with choosing new screening technologies that have the potential to be more beneficial than current strategies. Consistent with another analysis (Goldhaber-Fiebert et al, 2008), we found that HPV DNA testing in older women can be more cost-effective than cytology-only based strategies, for both vaccinated and unvaccinated women. For women who are not vaccinated, our primary analysis projected that the optimal strategy for primary screening involves cytology for younger women and HPV DNA testing with reflex LBC every 4 years for women aged 34 years and older. The algorithm requires three additional persistent HPV $+/$ Cyt - results 6 months apart, before colposcopy referral. For vaccinated women, the primary screening interval for older women could be extended to 6 years with the same follow-up for HPV + /Cyt- women. Our expanded secondary analysis concluded warranted the same primary screening intervals as the primary analysis but indicated that switching at an earlier age could further reduce lifetime costs while maintaining a similar reduction in the risk of cancer. We also found that it was rarely attractive to refer younger women with LSIL directly to colposcopy.

To our knowledge, this is the first analysis to evaluate the costeffectiveness of alternate screening strategies to prevent cervical cancer in Norway. We expand upon previous modelling studies, which look at primary HPV DNA testing in developed countries (Goldie et al, 2004, 2006; Sherlaw-Johnson and Philips, 2004; Kim et al, 2005; Bidus et al, 2006; Kulasingam et al, 2006; GoldhaberFiebert et al, 2008) to include new alternative triage strategies for older women who are HPV-positive, but cytology-negative. There is no consensus regarding how to optimally manage HPV-positive results to avoid over referral and unwarranted stress for women (Cuzick et al, 2006a) and the choice of management strategy for $\mathrm{HPV}+/$ Cyt - women may depend on other factors such as colposcopy resource constraints and preference to minimise false-positive results. The proposed management approach attempts to minimise the potential excess burden on resources and use a risk management strategy that identifies only the women at increased risk (i.e., those with persistent HPV infection) who have not developed dysplasia detectable by cytology. As we varied follow-up intervals of 6 or 12 months and number of persistent $\mathrm{HPV}+/ \mathrm{Cyt}-$ results required to prompt colposcopy, while holding all else constant, yielded relatively marginal changes to cancer risk reduction; our analysis suggests that it is rarely attractive to refer women to colposcopy after one additional $\mathrm{HPV}+/$ Cyt - result. We found that switching to primary HPV DNA testing at age 31 dominated switching at age 34 , one screening episode earlier than suggested by the Norwegian proposal. This is likely because the prevalence of high-risk HPV does not substantially change from 31 to 34 years, allowing women to capitalise on the additional benefit of HPV testing without the system incurring excess costs from a large number of transient infections. Determining the optimal switch age is inherently dependent on the natural history of HPV in older women.

Our analysis has clear limitations, many of which have been described previously (Goldhaber-Fiebert et al, 2007; Kim et al, 2007; Kim and Goldie, 2008). We chose to use a detailed simulation model that accommodates complex screening strategies and individual history at the expense of explicitly modelling herd immunity. In sensitivity analysis, we tried to simulate the indirect effects (herd immunity) that HPV vaccination may have on the incidence HPV-16, HPV-18 among unvaccinated women. We also chose not to include other HPV-related diseases. It would be expected that by preventing additional non-cervical cancers, we would see improved cost-effectiveness. We acknowledge the benefit of including quality-adjusted life years; however, cervical cancer health state utilities have not been published in Norway, and we therefore elected to express our results as cost per YLS. Cost per quality-adjusted life year ratios would likely yield more 
attractive ICERs and, therefore, we expect our cost per YLS to be a more conservative estimate.

Our cost estimates differed from those used for a previous analysis assessing the cost-effectiveness of the HPV vaccination in the context of the current screening programme in Norway (Dasbach et al, 2008). There have been certain disease-specific DRG updates (i.e., gynaecological brachytherapy; Norwegian Directorate of Health, 2010) since the publication, which help explain much of the difference in cancer treatment costs. The rank ordering of the results were stable when we varied the cancer treatment costs in our model from 50 to $200 \%$ of their base case values. We have also chosen to include direct non-medical costs, such as transportation and productivity loss directly attributable to screening and treatment. Norwegian wages are among the highest in the world and significantly contribute to the economic costs associated with screening and treatment. Through sensitivity analyses, we found that our main conclusion, with respect to screening interval for vaccinated and unvaccinated women, were robust to most cost assumptions; results were influenced only when doubling the costs associated with the primary office visit.

One limitation of the proposed strategy, which requires repeated follow-up of HPV + /Cyt- women before colposcopy referral, is the potential for loss-to-follow-up. Norwegian women are more likely to ignore recommendations to follow-up equivocal and lowgrade results compared with those indicating a high-grade lesion (Nygard et al, 2006). If the importance of continuing to follow-up an HPV + /Cyt - negative result is not communicated adequately to women, the additional sensitivity of HPV testing could be eroded. We did not evaluate whether this affects the optimal strategy, but it should be considered as a potential drawback of this particular screening algorithm.

The optimal strategies identified by this analysis will require a comprehensive and dynamic system, which can alert women according to their individual screening needs. More complex and tailored screening algorithms will be more difficult to understand, not only for women, but also for clinicians, who are responsible for explaining and implementing strategies. Extensive monitoring of the coverage, compliance, resource use, and outcome variables is also crucial in order to allow the public health officials to identify caveats and areas that are in need of improvement. Models can never replace true-life evaluation and as data accumulate, our model can be refined and revised.

\section{REFERENCES}

Aas E (2009) Pecuniary compensation increases participation in screening for colorectal cancer. Health Econ 18(3): 337-354

Arbyn M, Sasieni P, Meijer CJLM, Clavel C, Koliopoulos G, Dillner J (2006) Clinical applications of HPV testing: a summary of meta-analyses. Vaccine 24: $78-89$

Bidus MA, Maxwell GL, Kulasingam S, Rose GS, Elkas JC, Chernofsky M, Myers ER (2006) Cost-effectiveness analysis of liquid-based cytology and human papillomavirus testing in cervical cancer screening. Obstet Gynecol 107(5): $997-1005$

Bray F, Loos AH, McCarron P, Weiderpass E, Arbyn M, Moller H, Hakama M, Parkin DM (2005) Trends in cervical squamous cell carcinoma incidence in 13 European countries: changing risk and the effects of screening. Cancer Epidemiol Biomarkers Prev 14(3): $677-686$

Cancer Registry of Norway (2009) Annual Report 2008. Oslo. Available at: http://www.kreftregisteret.no/Global/Publikasjoner\%20og\%20rapporter/ Special\%20Issue/Special_Issue_Cancer_in_Norway_2009.pdf (accessed 6 January 2011)

Cancer Registry of Norway (2011) Cancer in Norway 2009. Special Issue: Cancer Screening in Norway. Oslo. Available at: http://www.kreftregisteret. no/Global/Publikasjoner\%20og\%20rapporter/Special\%20Issue/Special Issue_Cancer_in_Norway_2009.pdf (accessed 6 January 2011)

\section{CONCLUSION}

Our objective was to provide quantitative insight to policy makers about the trade-offs between different screening strategies, which use new screening technology in the context of HPV vaccination. We highlight the importance of alternative screening strategies that are conditional on vaccination status and age. The optimal strategies for vaccinated women determined by this analysis are very similar to the strategy that has been proposed for pilot testing in Norway (Cancer Registry of Norway, 2011). We shed light on the potential benefits of switching to HPV DNA testing at an earlier age and considering different screening recommendations for those women who have not been vaccinated. Given a costeffectiveness threshold of $\$ 83000$, it may be more efficient to screen unvaccinated women, more frequently than those women who were vaccinated during adolescents. We conclude that in Norway, strategies involving a switch to primary HPV testing in older women is expected to be cost-effective compared with the current cytology-based screening programme.

\section{ACKNOWLEDGEMENTS}

We are grateful for the contributions of Mari Nygaard, Steinar Thoresen, Leena Kiviluoto, Stephen Resch, Gry Skare, The Norwegian Cancer Registry, and the entire cervical cancer prevention team at the Center for Health Decision Science (Harvard School of Public Health). This work was supported in part by the University of Oslo, The Norwegian Cancer Society (nr: 634201-2010); JDO, SS, and JJK are funded in part by the US National Cancer Institute (R01 CA93435) and the Bill and Melinda Gates Foundation (30505) for related work in developing countries.

\section{Disclaimer}

Our work was independent of the funders and the funding sources had no involvement in the study design or conduct of the study; collection, management, analysis or interpretation of the data; or preparation, review or approval of the manuscript.

Supplementary Information accompanies the paper on British Journal of Cancer website (http://www.nature.com/bjc)
Cuzick J, Clavel C, Petry KU, Meijer CJLM, Hoyer H, Ratnam S, Szarewski A, Birembaut P, Kulasingam S, Sasieni P, Iftner T (2006a) Overview of the European and North American studies on HPV testing in primary cervical cancer screening. Int J Cancer 119(5): 1095-1101

Cuzick J, Mayrand MH, Ronco G, Snijders P, Wardle J (2006b) New dimensions in cervical cancer screening. Vaccine 24: 90-97

Dasbach EJ, Largeron N, Elbasha EH (2008) Assessment of the costeffectiveness of a quadrivalent HPV vaccine in Norway using a dynamic transmission model. Expert Rev Pharmacoecon Outcomes Res 8(5): 491 - 500 Dillner J, Rebolj M, Birembaut P, Petry KU, Szarewski A, Munk C, de Sanjose S, Naucler P, Lloveras B, Kjaer S, Cuzick J, van Ballegooijen M, Clavel C, Iftner T (2008) Long term predictive values of cytology and human papillomavirus testing in cervical cancer screening: joint European cohort study. BMJ 337(7676): doi. 10.1136/bmj.a1754

Federal Reserve (2011) Historical Rates for the Norwegian Krone. Available at: http://www.federalreserve.gov/RELEASES/H10/Hist/dat00_ no.htm (accessed 13 June 2011)

Franco EL (2003) Chapter 13: Primary screening of cervical cancer with human papillomavirus tests. J Natl Cancer Inst Monogr 2003(31): 89-96 Goldhaber-Fiebert JD, Stout NK, Ortendahl J, Kuntz KM, Goldie SJ, Salomon JA (2007) Modeling human papillomavirus and cervical cancer in the United States for analyses of screening and vaccination. Popul Health Metr 5: 11 
Goldhaber-Fiebert JD, Stout NK, Salomon JA, Kuntz KM, Goldie SJ (2008) Cost-effectiveness of cervical cancer screening with human papillomavirus DNA testing and HPV-16,18 vaccination. J Natl Cancer Inst 100(5): 308 - 320

Goldie SJ (2003) Chapter 15: Public health policy and cost-effectiveness analysis. J Natl Cancer Inst Monogr 2003(31): 102-110

Goldie SJ, Kim JJ, Myers E (2006) Cost-effectiveness of cervical cancer screening. Vaccine 24: $164-170$

Goldie SJ, Kim JJ, Wright TC (2004) Cost-effectiveness of human papillomavirus DNA testing for cervical cancer screening in women aged 30 years or more. Obstet Gynecol 103(4): 619-631

Hakama M, Hristova L (1997) Effect of screening in the Nordic cancer control up to the year 2017. Acta Oncol 36(2): 119-128

Kim JJ, Goldie SJ (2008) Health and economic implications of HPV vaccination in the United States. $N$ Engl J Med 359(8): $821-832$

Kim JJ, Kuntz KM, Stout NK, Mahmud S, Villa LL, Franco EL, Goldie SJ (2007) Multiparameter calibration of a natural history model of cervical cancer. Am J Epidemiol 166(2): $137-150$

Kim JJ, Wright TC, Goldie SJ (2005) Cost-effectiveness of human papillomavirus DNA testing in the United Kingdom, The Netherlands, France, and Italy. J Natl Cancer Inst 97(12): 888-895

Kulasingam SL, Myers ER, Lawson HW, McConnell KJ, Kerlikowske K, Melnikow J, Washington AE, Sawaya GF (2006) Cost-effectiveness of extending cervical cancer screening intervals among women with prior normal pap tests. Obstet Gynecol 107(2): $321-328$

Molden T, Kraus I, Karlsen F, Skomedal H, Hagmar B (2006) Human papillomavirus E6/E7 rnRNA expression in women younger than 30 years of age. Gynecol Oncol 100(1): 95-100

Molden T, Kraus I, Karlsen F, Skomedal H, Nygard JF, Hagmar B (2005) Comparison of human papillomavirus messenger RNA and DNA detection: a cross-sectional study of 4136 women $<30$ years of age with a 2-year follow-up of high-grade squamous intraepithelial lesion. Cancer Epidemiol Biomarkers Prev 14(2): 367-372

Munoz N, Bosch FX, de Sanjose S, Herrero R, Castellsague X, Shah KV, Snijders PJ, Meijer CJ, International Agency for Research on Cancer Multicenter Cervical Cancer Study Group (2003) Epidemiologic classification of human papillomavirus types associated with cervical cancer. N Engl J Med 348(6): 518-527

Norwegian Directorate of Health (2007) Health Effects of Socio-Economic Analyses. Available at: http://www.helsedirektoratet.no/vp/multimedia/ archive/00020/IS-1435_20969a.pdf (accessed on 6 January 2011)

Norwegian Directorate of Health (2010) Activity-based funding 2010/2011. Available at: http://www.helsedirektoratet.no/finansieringsordninger/ regelverk_innsatsstyrt_finansiering_isf_2011_78057 (accessed 10 December 2010)
Norwegian Finance Department (2005) Guideline for Economic Analysis. Available at: http://www.regjeringen.no/upload/kilde/fin/reg/2005/0029/ ddd/pdfv/266324-veileder_i_samfunnsok_analyse_trykket.pdf (accessed 6 January 2011)

Norwegian Medical Association (2010a) Guidelines for Cervical Cancer. Available at: http://www.legeforeningen.no/id/153817.0 (accessed 11 November 2010)

Norwegian Medical Association (2010b) Normal tariff for private general practice 2010-2011. Available at: www.legeforeningen.no/normaltariff/ Fastlegetariff_2010.pdf (accessed 1 November 2010)

Norwegian Medical Association (2010c) Normal tariff for private specialist practice 2010-2011. Available at: www.legeforeningen.no/normaltariff/ Normaltariff_2010.pdf (accessed 10 November 2010)

Norwegian Medicines Agency (2010) Reimbursement report: HPV-Vaccine Gardisil for prevention of HPV infection, cervical cancer and genital warts. Available at: http://www.legemiddelverket.no/upload/144901/ 09-16212-8\%20RAPPORT\%201622714_underskrevet.pdf (accessed 1 May 2011)

Nygard JF, Nygard M, Skare GB, Thoresen SO (2006) Pap smear screening in women under 30 in the Norwegian Coordinated Cervical Cancer Screening Program, with a comparison of immediate biopsy vs Pap smear triage of moderate dysplasia. Acta Cytologica 50(3): $295-302$

Peto J, Gilham C, Fletcher O, Matthews FE (2004) The cervical cancer epidemic that screening has prevented in the UK. Lancet 364(9430): $249-256$

Sherlaw-Johnson C, Philips Z (2004) An evaluation of liquid-based cytology and human papillomavirus testing within the UK cervical cancer screening programme. Br J Cancer 91(1): 84-91

Sherman ME (2003) Chapter 11: Future Directions in Cervical Pathology. J Natl Cancer Inst Monogr 2003(31): $72-79$

Solomon D (2003) Chapter 14: Role of Triage Testing in Cervical Cancer Screening. J Natl Cancer Inst Monogr 2003(31): 97-101

Statistics Norway (2011) Available at: http://www.ssb.no/english/ (accessed 10 January 2011)

Stinnett AA, Paltiel AD (1997) Estimating CE ratios under second-order uncertainty: the mean ratio versus the ratio of means. Med Decis Making 17(4): $483-489$

World Health Organization (2003) World Health Survey. Available at: http://www.ssb.no/whs_en/ (accessed 5 January 2011)

Wright TC, Massad S, Dunton CJ, Spitzer M, Wilkinson EJ, Solomon D (2007) 2006 consensus guidelines for the management of women with abnormal cervical cancer screening tests. Am J Obstet Gynecol 197(4): $346-355$

This work is published under the standard license to publish agreement. After 12 months the work will become freely available and the license terms will switch to a Creative Commons Attribution-NonCommercial-Share Alike 3.0 Unported License. 